\title{
Mind Wandering: Mechanism, Function, and Intervention
}

\author{
Zhun Gong1,2*, Yaru Ding ${ }^{1}$ \\ ${ }^{1}$ Department of Psychology, Teachers College, Qingdao University, Qingdao, China \\ ${ }^{2}$ Qingdao Psychological and Mental Health Research Institute, Qingdao University, Qingdao, China \\ Email: *gongzhun2001@163.com
}

How to cite this paper: Gong, Z., \& Ding, Y. R. (2018). Mind Wandering: Mechanism, Function, and Intervention. Psychology, 9, 2662-2672.

https://doi.org/10.4236/psych.2018.912152

Received: October 13, 2018

Accepted: November 24, 2018

Published: November 27, 2018

Copyright (c) 2018 by authors and Scientific Research Publishing Inc. This work is licensed under the Creative Commons Attribution International License (CC BY 4.0).

http://creativecommons.org/licenses/by/4.0/

\section{cc) (i) Open Access}

\begin{abstract}
Mind wandering is an important part of the stream of consciousness, which allows the stream of consciousness to continue when the individual does not process external stimuli or does tasks. The neural mechanism of mind wandering is the current research hotspot. This study involves the brain's default network and two theories of different positions, one is the theory of executive control, and the other is the theory of executive control failure. The study discusses adaptive functioning of mind wandering, especially in terms of creative problem solving. Finally, there is a discussion of how to reduce the negative effects of mind wandering-mindfulness training.
\end{abstract}

\section{Keywords}

Mind Wandering, Neural Mechanisms, Default Network, Executive Control Theory, Mindfulness

\section{Introduction}

In the fields of cognitive neuroscience and psychology, there has been a wave of interest in the study of mind wandering. Every one of us should have had the experience, for example, when you were working on a document editing job, did you ever get the idea that your mind had drifted away to eat at night? Or you may find that you can't remember the steps and principles that the teacher has just explained. This situation, for different studies, has different definitions. In this article, we call this phenomenon "mind wandering". In other words, mind wandering referred to a shift in the contents of thought away from an ongoing task and/or from events in the external environment to self-generated thoughts and feelings.

People will find that sometimes individuals can let their ideas flow freely, 
away from their activities. And it happens quite frequently every day. Previous experimental studies have shown that adults have mental wandering about 30 to 50 percent of the time when they are awake every day (Andrews-Hanna et al., 2014). So what is the mechanism by which mind wandering occurs. There is a combination of executive control and executive control failure (McVay \& Kane, 2010) that explains the mind wandering. At the same time, a large number of studies on the cognitive neural mechanism show that the default mode network is one of the important factors for the generation of mind wandering. Default mode network: a network of brain regions involving the medial surface of the cortex that is engaged by the sort of thinking that occurs during mind wandering (e.g., the future, the self, other people) and is active during periods of mind wandering (Smallwood \& Schooler, 2015). In addition, many studies have focused on the loss of task performance and even psychological distress caused by mind wandering (Mooneyham \& Schooler, 2013). So we try to focus on the other side of mind wandering-its functional role and how it can be used to creatively solve problems.

Finally, based on previous studies, we will explain how mindfulness can be used to reduce the negative effects of mind wandering in our daily lives.

\section{Mental and Neural Mechanisms of Mind Wandering}

\subsection{Default Mode Network}

Although mind wandering accounts for a large proportion of our waking lives, its neural basis and relationship to sustained behavior remain controversial. In one of the most interesting areas of neuroscience in the past two decades, there is a study that researchers observed that certain areas of the brain begin to function, while these areas are less in need of external tasks. This brain region is the "default mode network" (Shulman et al., 1997; Raichle et al., 2001; Gusnard \& Raichle, 2001).

The default mode network is a network composed of several brain regions that are interconnected and maintain healthy metabolic activity while the brain is at rest. The posterior cingulate gyrus and medial prefrontal cortex form the core of the default mode network, and also interact with subnetworks including the medial temporal leaf system and the dorsal subsystem (Andrews-Hanna et al., 2014). The core of the posterior cingulate gyrus and medial prefrontal cortex is often involved in self-reference, or autobiographical memory; The inner subsystem is usually involved in the process of the plot, such as the recognition of the scene or simulation; The dorsal subsystem is involved in social processes, such as analyzing the behavioral intention of others or reviewing some social common sense.

A growing number of research topics are discussing the role of default mode networks in internalizing or self-generated thoughts. The use of self-reported mind wandering suggests that the individual's self-generated thoughts at this time typically consist of three parts: reminiscing about the past, planning for the 
future, and other thoughts and experiences about the individual.

Spontaneous thought is divided into two situations: the first is as part of a task, where the individual must make a decision depending on the internal representation to understand the stimulus and produce the answer to the problem by reconstructing or imagining the situation. The second type occurs independently of external tasks, such as when individuals are daydreaming or when they are performing tasks (Andrews-Hanna et al., 2014).

Therefore, research has raised the possibility that the mental content of self-generated mind wandering is the product of the episodic memory system (Smallwood \& Schooler, 2015). In addition, studies using FMRI and empirical sampling have provided strong theoretical support for the hypothesis of episodic memory (Christoff et al., 2009). It is proved that the default mode network is involved in the thoughts experienced by an individual during mind wandering. When an individual's task is to imagine another place or time (Addis et al., 2012) or when he thinks about self-correlation (Kelley et al., 2002), the regional activity of the default mode network is significantly enhanced.

Therefore, we can consider the process of mind wandering as the opposite of external perception. In other words, in the resting state, the core area of the default mode network is negatively correlated with the core area of the brain involved in the process of external perception (such as the occipital cortex) (Vincent et al., 2006). Other studies have also shown that the default mode network is characterized by increased activity during the period of task-independent thinking (Allen et al., 2013). So we can infer that mind wandering is important to our brain. The seemingly pointless "daydreams" or "mind-wandering" that we generate in our daily lives are then integrated by the default mode network, which facilitates future behavioral choices.

\subsection{Executive Control Theory}

The default mode network is not alone. Besides the default network, it is also associated with the neural mechanism of mind wandering. Besides the default network, it is also associated with the behavioral theory of task irrelevant thoughts and the relationship of executive control resources. Studies have emphasized the dynamics of the default network and its interaction with the executive control system in the management of internal thoughts (Andrews-Hanna et al., 2014). Executive control function refers to the psychological process in which individuals have conscious control over their thoughts and behaviors. Mind wandering consumes execution resources (Smallwood \& Schooler, 2006), during which we are concerned with pulling our execution functions away from the current external tasks and applying them to task independent, internally generated stimulus content. That is to say, during the mind wandering, the resources to perform the control function are not all consumed in the external task, but some of them are used in the spontaneous thought. At the same time, other studies showed that when subjects reported that they were not aware of their mind 
wandering, their default network and executive network had higher neural engagement than when they were aware of their mind wandering (McVay \& Kane, 2010).

It can be seen from this that mind wandering is most obvious when individuals lack metacognition (Christoff et al., 2009). That is, in the absence of effective metacognitive monitoring, the brain regions responsible for mental locomotion are more likely to separate the executive resources from the current main tasks and allocate them to mental locomotion activities.

Christoff et al. (2009) found that some areas of the executive system (such as the anterior cingulate gyrus and dorsolateral prefrontal cortex) were also activated during mind wandering using empirical sampling and FMRI techniques. At present, behavioral and neuroimaging studies offer different perspectives on the contribution of the executive system to mind wandering. Behavioral research using empirical sampling (Teasdale et al., 1995; Smallwood \& Schooler, 2006; Kane et al., 2007) demonstrated that mind wandering is a complex psychological activity that often interferes with cognitively demanding tasks, suggesting that task processing overlaps with the executive system of the brain. In contrast, neuroimaging results focus on the default network (McKiernan et al., 2006; Mason et al., 2007). So far, neuroimaging studies have concluded that compared mind wandering, in under the condition of different tasks are highly familiar with tasks and "rest" conditions (It is known with a relatively high incidence of mind wandering related) with novel and requiring higher cognitive task (It is known is associated with a relatively low incidence of mind wandering) (Smallwood \& Schooler, 2006; Mason et al., 2007). In these comparisons, a related reduction in cognitive needs from activation to baseline may inhibit the participation of default network areas during mind wandering. So it turns out that the default network of the brain is called upon to engage in mind wandering.

Therefore all of these studies support the conclusion that mind wandering really requires the resource consumption of the executive system. Mind wandering varies with the difficulty of the task, and Smallwood and Schooler (2006) explain this finding as a trade-off between the execution of the task and the execution of unrelated thoughts. For example, as the rate of stimulus presentation increases in vigilance tasks and other aspects of difficulty as memory burdens or tasks increase, mind wandering decreases accordingly (Ruby et al., 2013). However, there is still a clear difference between mind wandering and typical executive control theory, that is, executive control emphasizes the obvious purpose in processing. However, in the state of mind wandering, the individual is often generated without paying attention (i.e. without metacognition).

Many experimental results support the theory of executive control of mind wandering. But some researchers don't agree on the exact relationship between mind wandering and executive control. So there's a lot of debate as to whether mind wandering reflects executive control or failure of executive control. Experimental studies have shown that mind wandering is caused by the failure of ex- 
ecutive control (Kane \& McVay, 2012), without consuming execution resources.

\subsection{Executive Control Failure Theory}

Mind wandering produced not only depends on external stimuli and inner clues to respond by the emergence of authigenic thought still depends on the executive control system is unable to process due to the Task irrelevant thoughts (Task-Unrelated thoughts). Klinger's current concerns combined with the assumption of executive control failure partly supports the idea that mind wandering is caused by executive control failure. The executive control system should play a role in controlling or preventing mind wandering. Therefore, mind wandering reflects the failure of the control system, possibly because the execution resources cannot be used for correct thought control (McVay \& Kane, 2010).

Previous research has shown that, if the assumption is made that executing resources are exhausted in the case of demanding tasks and alcohol overdose, and that mental wandering requires executing resources, then task-free thinking should be reduced in the case of excessive fatigue or drunkenness. However, Kane and McVay believed that the failure of the executive control system led to mind wandering. As the executive control system became tired or impaired due to alcohol, the resulting tasks should have more failures or errors, and mind wandering should occur more frequently. For the mechanism of mind wandering, Smallwood proposed to combine the theory of executive control and the hypothesis of executive control failure for interpretation and explanation. The relationship between mind wandering and executive control needs further exploration.

\section{The Adaptive Function of Mind Wandering Is about Creative Problem Solving}

As the research on mind wandering deepens, previous studies have paid more attention to the costs and costs of mind wandering, but more and more recent studies have noticed that mind wandering also has some positive adaptive function.

In particular, how to solve problems creatively, how to improve students' creativity and how to improve work performance by promoting decision-making. About how to solve the problem, creatively Baird et al., set up an experiment, the participants in the setting time for common everyday objects (e.g., bricks) named as much as possible the purpose of (called the unusual use of task (UUT)) (Mrazek et al., 2012a), the rest time between two UUT, for don't need to be highly focused tasks or task difficulty is relatively low, the mind is among the highest of shifting frequency.

In the meantime, the more content participants thought about, the more ideas they came up with about the object's purpose. In the past, some studies have shown that mind wandering does not seem to improve the performance of projects that have not been encountered before. Baird et al. believe that instead 
of playing a general role in improving creativity, it is better to provide creative opportunities to come up with new solutions to problems that have been encountered before (Mrazek et al., 2012a).

It is obvious, therefore, that the conditions for maximum mental mobility are also most conducive to creative problem solving, although it should also be noted that the performance of repetitive creativity problems and new creativity problems is positively correlated with the mind wandering tendency of individuals measured by image process inventory (Ruby et al., 2013). In this study, Braid et al. found a relationship between individuals' tendency to drift their minds and their performance on abnormal use tests, an indicator of divergent thinking (Smallwood \& Andrews-Hanna, 2013; Smallwood \& Schooler, 2015; Kane et al., 2017). In addition, there is the application of the theory of "hatching effect", that is, by diverting attention from a headache-inducing problem, thereby temporarily relieving the individual from his conscious effort to solve the problem. Mind wandering can shift one's problem-solving efforts to unconscious processing, which has been theorized to facilitate new associations and combinations of existing knowledge (Schooler et al., 2011; Mooneyham \& Schooler, 2013; Zedelius \& Schooler, 2015; Dane, 2018).

A mind-wandering environment (i.e., engaging in non-demanding tasks or less demanding tasks) has more potential benefits than those that require constant external attention or idle breaks (Zedelius \& Schooler, 2015), involved in the study of creative problem solving to mind wandering research can indirectly reflect the Wallace (Wallas) four stages theory (namely the study of creative thinking, important model, the model that creative thinking stage including preparation, gestation period, anacreontic, validation) in the brain mechanism of gestation period, with the default mode network have a close relationship.

Existing research on creative thinking has also shown that mind wandering contributes to the performance of individual creative tasks. The gestation period in the four-stage theory indicates that the brain region associated with the default mode network will be activated, and the gestation period is needed to trigger the emergence of Epiphany. So we can conclude that mind wandering does promote creative thinking. As for the task performance of mind wandering in organizations, as the researchers have observed, mind wandering can promote creativity (Mooneyham \& Schooler, 2013).

Creativity is a key input in many job performances in today's world (Dane, 2018). In addition to the incubation effect described above, there is another way in which the creative benefits of mind wandering can be seen. In some mind-wandering plots, the individual can generate possibilities by free sliding (Dane, 2018). In some cases, interesting possibilities created by mind wandering can constitute creative ideas, that is, ideas that are both new and useful. In fact, it is often found in study or work that if we stop focusing on the result of things and turn to the freedom and flexibility of exploring or constructing "methods" to solve problems (Dane, 2018), there will be interesting thinking. 
The interesting possibilities of mind wandering can promote creativity by facilitating problem discovery (Robison \& Unsworth, 2018). This means "inventing" a problem that is worth solving. Usually, we mean the discovery of a problem in the presence of a problem (for example, the discovery of a faulty steam engine that needs to be repaired in time).

\section{Mindfulness Training Interventions for Mind Wandering}

Mindfulness training has been widely used in our daily life, such as meditation training, yoga, etc. One view defines mindfulness as persistent unbiasedness (Brown \& Harkins, 2016; Zanesco et al., 2016) and multi-factor mindfulness not only emphasizes the knowledge of current experience, but also emphasizes curiosity, openness and acceptance (Mrazek et al., 2012b; Rahl et al., 2017). Mindfulness is purposeful and conscious. It pays attention to and perceives everything in the present, and does not make any judgments, any analysis, or any reaction to the present. It simply observes it and pays attention to it. Mindfulness has its roots in Eastern contemplative traditions. In fact, mindfulness has been called the "heart" of Buddhist meditation. Mindfulness, however, is more than meditation. It is "inherently a state of consciousness" which involves consciously attending to one's moment-to-moment experience (Brown \& Ryan, 2003). Although there are differences of opinion among the studies, the researchers agree that sustained attention is essential to mindfulness. Given that mindfulness and mind wandering seem to be opposing constructs for the ability to remain undisturbed, it has become a current focus to examine whether mindfulness training can reduce mental wandering. First, contrary to mindfulness, mind wandering requires the ability to avoid distractions, characterized by the interruption of task focus (TUT) (Mooneyham \& Schooler, 2016; Pachai et al., 2016; Wu et al., 2016; Zanesco et al., 2016).

Many behavioral markers of mind wandering have a distinct trait, such as absent-minded forgetting (Cheyne et al., 2006) and paying little attention to vocabulary or language features when reading. In addition, underlying studies related to events suggest that mind wandering is characterized by reduced awareness of task stimuli and external environment (Schooler et al., 2011; Zanesco et al., 2016). As a result, the ability to focus on a task seems directly counter to the tendency for attention to linger on issues unrelated to the task. So we can assume that the place where mindfulness ends is the beginning of mind wandering.

Studies have shown that mindfulness training can improve participants' reading comprehension scores on the GRE test and their working memory ability (Mrazek et al., 2013). Studies have also shown that mindfulness training is protective for mind wandering in individuals with anxiety. Meditation prevents thoughts from increasing over time and improves performance interruptions during tasks. At the same time, the researchers also found that the meditative intervention can promote the shift of attention from the internal world to the 
external world (Xu et al., 2017).

Researchers have shown that mindfulness training, regardless of its intensity, can significantly reduce mind wandering and improve performance in the general population (Mrazek et al., 2013). However, the results of this study are consistent with those of other studies (Zedelius \& Schooler, 2015), which suggest that mindfulness training can only prevent mental wandering from adversely affecting individuals who suffer from highly negative effects.

\section{Future Research Issues}

The specific term "mind wandering" has increased in recent years, with definitions like "mind-wandering", "daydreaming", and "task-free thinking" appearing more frequently in studies before 2002. We can see that long-term studies have looked at the negative effects of mental wandering (e.g. Mind wandering leads to traffic accidents). But, as mentioned above, there's also research on how mental wandering plays its adaptive role in increasing creativity. Future research should focus on exploring the potential adaptive function of mind wandering. Even if studies have found the potential function of mind wandering, there is no evidence to support it as well as the negative effects of mind wandering. What's more, what's most interesting is the brain mechanisms of mind wandering, what do individuals think when they think about their thoughts? How does the default mode network interact with other large-scale brain systems while maintaining internal thinking?

Regardless of the method used to measure mind wandering, the researchers' point is simply to compare and contrast changes in brain areas during mind wandering versus changes in non-mind wandering. However, recently Christoff et al. (2016) have made a distinction between conscious mind wandering and unconscious mind wandering from the perspective of different mental states (such as daydreaming, mind wandering, creative thinking, goal-directed thinking, and compulsive thinking) and proposed a new theoretical framework.

All these reveal that future research should not only study the unconscious mind wandering, but also pay attention to the mechanism, effect and influence of conscious mind wandering. Other researchers have found that people tend to experience restlessness during periods of inattention or mind wandering. One study examined whether individual differences in inattention and mind wandering in daily life were associated with spontaneous and involuntary movements (i.e., restlessness) (Christoff et al., 2009; Mooneyham \& Schooler, 2013; Smallwood \& Andrews-Hanna, 2013). Further needs to understand individual differences in mind wandering, including negative characteristics (e.g., various mental disorders) and positive characteristics (e.g., creativity) in relation to them.

Finally, we need to identify reliable behavioral and physiological measures that can indicate that mind wandering begins and cancels, rather than relying on individual self-reporting. By making more accurate judgments of mind wander- 
ing, develop more techniques to minimize mind wandering (at inopportune times) and further observe the effects of mindfulness training.

\section{Conclusion}

With a growing interest in mind wandering, a con-comitant increase in attention directed toward rigorous research in this area is needed. We have introduced comprehensively a perspective on the role of default mode network and different executive control theories. A lot of research has shown activation of default mode network regions during mind wandering. However, there is still controversy about whether it is due to the execution of control to consume resources or the execution of control failure. More researchers are still inclined to approve the idea of controlling consumption of resources. A new perspective on the mechanism of mind wandering thinks that the theory of executive control and the hypothesis of executive control failure are combined for explanation.

Moreover, we introduced the adaptive function of mind wandering. In terms of creativity, mind wandering has a huge advantage. We often think that mind wandering has great drawbacks in learning and work, but as the research progresses, its benefits are slowly being explored. And mindfulness can reduce the mind wandering and change the performance of the general population which has been confirmed by research.

\section{Acknowledgements}

This research is supported by grants from the National Social Science Fund of China, Qingdao Research Institute of Psychology and Mental Health project and Ministry of Education "Human Factors and Ergonomics" Production and Education Project awarded to the first author. This research provides theoretical support for the above projects. Project numbers are 14CGL073, QPMHI201803 and 201702145005.

\section{Conflicts of Interest}

The authors declare no conflicts of interest regarding the publication of this paper.

\section{References}

Addis, D. R. et al. (2012). Routes to the Past: Neural Substrates of Direct and Generative Autobiographical Memory Retrieval. NeuroImage, 59, 2908-2922.

Allen, M. et al. (2013). The Balanced Mind: The Variability of Task-Unrelated Thoughts Predicts Error Monitoring. Frontiers in Human Neuroscience, 7, 743.

Andrews-Hanna, J. R. et al. (2014). The Default Network and Self-Generated Thought: Component Processes, Dynamic Control, and Clinical Relevance. Annals of the New York Academy of Sciences, 1316, 29-52. https://doi.org/10.1111/nyas.12360

Brown, A. J., \& Harkins, S. G. (2016). Threat Does Not Make the Mind Wander: Reconsidering the Effect of Stereotype Threat on Mind-Wandering. Motivation Science, 2, 85-96. 
Brown, K. W., \& Ryan, R. M. (2003). The Benefits of Being Present: Mindfulness and Its Role in Psychological Well-Being. Journal of Personality and Social Psychology, 84, 822-848. https://doi.org/10.1037/0022-3514.84.4.822

Cheyne, J. A. et al. (2006). Absent-Mindedness: Lapses of Conscious Awareness and Everyday Cognitive Failures. Consciousness and Cognition, 15, 578-592.

Christoff, K. et al. (2009). Experience Sampling during fMRI Reveals Default Network and Executive System Contributions to Mind Wandering. Proceedings of the National Academy of Sciences, 106, 8719-8724.

Christoff, K. et al. (2016). Mind-Wandering as spontaneous Thought: A Dynamic Framework. Nature Reviews Neuroscience, 17, 718-731.

https://doi.org/10.1038/nrn.2016.113

Dane, E. (2018). Where Is My Mind? Theorizing Mind Wandering and Its Performance-Related Consequences in Organizations. Academy of Management Review, 43, 179-197.

Gusnard, D. A., \& Raichle, M. E. (2001). Searching for a Baseline: Functional Imaging and the Resting Human Brain. Nature Reviews, 2, 685-694.

Kane, M. J. et al. (2007). For Whom the Mind Wanders, and When an Experience-Sampling Study of Working Memory and Executive Control in Daily Life. Psychological Science, 18, 614-621.

Kane, M. J. et al. (2017). A Combined Experimental and Individual-Differences Investigation into Mind Wandering during a Video Lecture. Journal of Experimental Psychology: General, 146, 1649-1674.

Kane, M. J., \& McVay, J. C. (2012). What Mind Wandering Reveals about Executive-Control Abilities and Failures. Current Directions in Psychological Science, 21, 348-354.

Kelley, W. M. et al. (2002). Finding the Self? An Event-Related fMRI Study. Journal of Cognitive Neuroscience, 14, 785-794. https://doi.org/10.1162/08989290260138672

Mason, M. F. et al. (2007). Wandering Minds: The Default Network and Stimulus-Independent Thought. Science, 315, 393-395.

McKiernan, K. A. et al. (2006). Interrupting the "Stream of Consciousness": An fMRI Investigation. NeuroImage, 29, 1185-1191.

McVay, J. C., \& Kane, M. J. (2010). Does Mind Wandering Reflect Executive Function or Executive Failure? Comment on Smallwood and Schooler (2006) and Watkins (2008). Psychological Bulletin, 136, 188-197.

Mooneyham, B. W., \& Schooler, J. W. (2013). The Costs and Benefits of Mind-Wandering: A Review. Canadian Journal of Experimental Psychology, 67, 11-18. https://doi.org/10.1037/a0031569

Mooneyham, B. W., \& Schooler, J. W. (2016). Mind Wandering Minimizes Mind Numbing: Reducing Semantic-Satiation Effects through Absorptive Lapses of Attention. Psychonomic Bulletin \& Review, 23, 1273-1279. https://doi.org/10.3758/s13423-015-0993-2

Mrazek, M. D. et al. (2012a). The Role of Mind-Wandering in Measurements of General Aptitude. Journal of Experimental Psychology: General, 141, 788-798. https://doi.org/10.1037/a0027968

Mrazek, M. D. et al. (2012b). Mindfulness and Mind-Wandering: Finding Convergence through Opposing Constructs. Emotion, 12, 442-448. https://doi.org/10.1037/a0026678

Mrazek, M. D. et al. (2013). Mindfulness Training Improves Working Memory Capacity and GRE Performance While Reducing Mind Wandering. Psychological Science, 24, 
776-781. https://doi.org/10.1177/0956797612459659

Pachai, A. A. et al. (2016). The Mind That Wanders: Challenges and Potential Benefits of Mind Wandering in Education. Scholarship of Teaching and Learning in Psychology, 2, 134-146. https://doi.org/10.1037/st10000060

Rahl, H. A. et al. (2017). Brief Mindfulness Meditation Training Reduces Mind Wandering: The Critical Role of Acceptance. Emotion, 17, 224-230. https://doi.org/10.1037/emo0000250

Raichle, M. E. et al. (2001). A Default Mode of Brain Function. PNAS, 98, 676-682. https://doi.org/10.1073/pnas.98.2.676

Robison, M. K., \& Unsworth, N. (2018). Cognitive and Contextual Correlates of Spontaneous and Deliberate Mind-Wandering. Journal of Experimental Psychology: Learning, Memory, and Cognition, 44, 85-98. https://doi.org/10.1037/xlm0000444

Ruby, F. J. et al. (2013). How Self-Generated thought Shapes Mood-The Relation between Mind-Wandering and Mood Depends on the Socio-Temporal Content of Thoughts. PLoS ONE, 8, e77554. https://doi.org/10.1371/journal.pone.0077554

Schooler, J. W. et al. (2011). Meta-Awareness, Perceptual Decoupling and the Wandering Mind. Trends in Cognitive Sciences, 15, 319-326. https://doi.org/10.1016/j.tics.2011.05.006

Shulman, G. L. et al. (1997). Common Blood Flow Changes across Visual Tasks: II. Decreases in Cerebral Cortex. Journal of Cognitive Neuroscience, 9, 648-663. https://doi.org/10.1162/jocn.1997.9.5.648

Smallwood, J., \& Andrews-Hanna, J. (2013). Not All Minds That Wander Are Lost: The Importance of a Balanced Perspective on the Mind-Wandering State. Frontiers in Psychology, 4, 441. https://doi.org/10.3389/fpsyg.2013.00441

Smallwood, J., \& Schooler, J. W. (2006). The Restless Mind. Psychological Bulletin, 132, 946-958. https://doi.org/10.1037/0033-2909.132.6.946

Smallwood, J., \& Schooler, J. W. (2015). The Science of Mind Wandering: Empirically Navigating the Stream of Consciousness. Annual Review of Psychology, 66, 487-518. https://doi.org/10.1146/annurev-psych-010814-015331

Teasdale, J. D. et al. (1995). Stimulus-Independent Thought Depends on Central Executive Resources. Memory \& Cognition, 23, 551-559.

Vincent, J. L. et al. (2006). Coherent Spontaneous Activity Identifies a Hippocampal-Parietal Memory Work. Journal of Neuropsychology, 96, 3517-3531.

Wu, G. et al. (2016). Mind Wandering While Reading: Theoretical Assumptions and Factors. Advances in Psychological Science, 24, 196. https://doi.org/10.3724/SP.J.1042.2016.00196

$\mathrm{Xu}, \mathrm{M}$. et al. (2017). Mindfulness and Mind Wandering: The Protective Effects of Brief Meditation in Anxious Individuals. Consciousness and Cognition, 51, 157-165. https://doi.org/10.1016/j.concog.2017.03.009

Zanesco, A. P. et al. (2016). Meditation Training Influences Mind Wandering and Mindless Reading. Psychology of Consciousness: Theory, Research, and Practice, 3, 12-33. https://doi.org/10.1037/cns0000082

Zedelius, C. M., \& Schooler, J. W. (2015). Mind Wandering “Ahas” versus Mindful Reasoning: Alternative Routes to Creative Solutions. Frontiers in Psychology, 6, 834. https://doi.org/10.3389/fpsyg.2015.00834 